\title{
Robust Topological Features for Deformation Invariant Image Matching *
}

\author{
Edgar Lobaton ${ }^{1}$, Ram Vasudevan $^{2}$, Ron Alterovitz $^{1}$, and Ruzena Bajcsy ${ }^{2}$ \\ ${ }^{1}$ Department of Computer Science \\ University of North Carolina, Chapel Hill \\ \{lobaton, ron\}ecs. unc.edu \\ ${ }^{2}$ Department of Electrical Engineering and Computer Sciences \\ University of California, Berkeley \\ $\{$ ramv, bajcsy\}@eecs.berkeley.edu
}

\begin{abstract}
Local photometric descriptors are a crucial low level component of numerous computer vision algorithms. In practice, these descriptors are constructed to be invariant to a class of transformations. However, the development of a descriptor that is simultaneously robust to noise and invariant under general deformation has proven difficult. In this paper, we introduce the Topological-Attributed Relational Graph (T-ARG), a new local photometric descriptor constructed from homology that is provably invariant to locally bounded deformation. This new robust topological descriptor is backed by a formal mathematical framework. We apply T-ARG to a set of benchmark images to evaluate its performance. Results indicate that T-ARG significantly outperforms traditional descriptors for noisy, deforming images.
\end{abstract}

\section{Introduction}

Local photometric descriptors have found successful application in numerous areas such as object recognition [8], wide baseline matching [13], and image retrieval [10]. Traditionally these descriptors have been constructed in order to be invariant to a specific class of transformations while remaining robust to noise. In practice, most have focused on the development of descriptors that are invariant under affine transformations as this is what occurs when a viewpoint changes relative to a rigid object with locally planar regions. Unfortunately, this class of transformations is unable to encapsulate the class of continuous deformations

*This research is supported by the National Science Foundation under awards CCR-0325274, CNS-0953823, CRA-0937060, ECCS-0931437, IIS-0703787, IIS-0724681, IIS-0840399, and IIS-0905344 and the National Institute of Health under award R21EB011628.

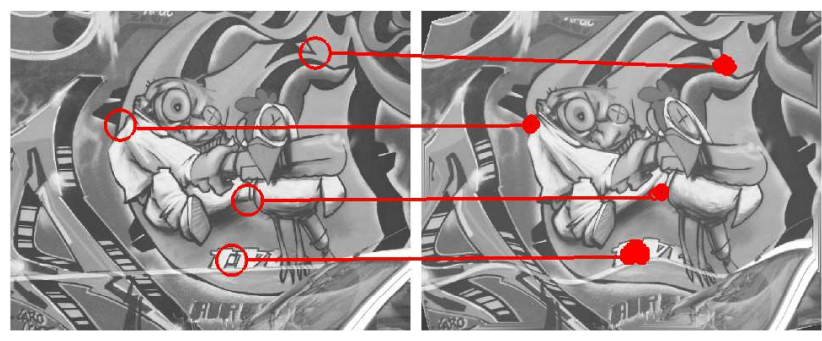

Figure 1. Sample Results. The image on the right is constructed after deforming the left image by $30 \%$ and the red circles connected by red lines correspond to matches according to the algorithm presented in this paper.

that describe how non-rigid objects transform, such as an animal moving its body or a cloth being folded. Observe that outside of occluding points, the class of continuous deformations is able to describe the transformation between pairs of views of the same scene or the evolution of a deforming object seen from the same view. Under deformation, it is well known that the appropriate invariant is a topological one, i.e. the number of connected components or holes. However, such invariants have two principal shortcomings: (1) they are not resilient to the presence of noise and (2) they tend not to be distinct.

In this paper, we propose a novel framework for building a topological descriptor that is invariant under locally bounded deformations and that addresses these two shortcomings. First, we make the topological invariants robust to noise by defining them in a local region over several inter-level sets of the intensity image. Second, we make the topological invariants distinct by describing the relational structure of nearby topological invariants. In so doing, we define a robust, distinct descriptor called the TopologicalAttributed Relational Graph or T-ARG. Fig. 1 illustrates the performance of our descriptor on a typical pair of images. 


\subsection{Related Work}

Due to space constraints, we refer the reader requiring an introduction to local descriptors to the comprehensive survey of the field by Mikolajczyk et al. [11]. They also provide a useful performance evaluation of several local descriptors including complex filters [12], gradient location and orientation histograms (GLOH) [11], shape context [1], scale invariant feature transform (SIFT) [9], spin image [4], and steerable filters [2]. In particular, SIFT and GLOH outperform all other descriptors. Our method can be loosely categorized with the so-called distribution based descriptors, like GLOH and SIFT, which use histograms to capture local image information. Importantly our method differs from these aforementioned approaches since it is invariant to all locally bounded deformations.

Others have attempted to develop local descriptors that are deformation invariant. The most promising such descriptor was developed by Ling et al. [5] who treat an intensity image as a surface embedded in 3D space, with intensity weighted relative to distance in the $x, y$ plane. They show that as this weight increases, geodesic distances on the embedded surface are less affected by image deformation. They use geodesic sampling to construct a descriptor called the geodesic-intensity histogram (GIH). The method, though interesting, is not resilient to the presence of noise, since noise in either image does not scale well with respect to the weighting parameter. Our method on the other hand is provably robust to the presence of noise since it operates over several inter-level sets of the intensity image simultaneously. Our new descriptor T-ARG is motivated by our recent work that defines a topological description of occlusions during deformation [7].

\subsection{Overview}

Our principal contributions are two-fold. First, in Section 3 we develop a notion of topological invariance under the assumption of locally bounded deformation. Second, in Section 4 we show how these topological invariants can be combined locally to define a descriptor that is distinct. The rest of the paper is organized as follows: Section 2 describes our imaging model and describes the type of properties we wish our descriptor to satisfy; Section 5 describes explicitly how to employ T-ARG to compare descriptors; Section 6 compares the performance of T-ARG to SIFT and GLOH using a precision versus recall metric; and Section 7 concludes the paper.

\section{Image Model}

In this section we introduce our imaging model and formalize the objectives of our work. Our analysis is done on grayscale images, but can be generalized to a multi-channel imaging modality in a straightforward manner. Suppose we are given two grayscale images $I_{0}, I_{1}: \Omega \rightarrow \mathbb{R}$ defined over an image domain $\Omega \subset \mathbb{R}^{2}$ and related by:

$$
I_{1}(x)=I_{0} \circ f(x)+p(x),
$$

where $p: \Omega \rightarrow \mathbb{R}$ is a scalar-valued function and $f: \Omega \rightarrow$ $\Omega$ is a homeomorphism that satisfies:

$$
\begin{aligned}
|p(x)| & \leq K_{p}, \\
h_{L}\left(\| x-x^{\prime}||\right) & \leq\left\|f(x)-f\left(x^{\prime}\right)\right\|, \text { and } \\
\left\|f(x)-f\left(x^{\prime}\right)\right\| & \leq h_{U}\left(\left\|x-x^{\prime}\right\|\right)
\end{aligned}
$$

for all $x, x^{\prime} \in \Omega$, where $h_{L}, h_{U}: \mathbb{R}^{+} \rightarrow \mathbb{R}^{+}$are monotonic increasing functions with $h_{L}(\rho) \leq h_{U}(\rho)$ for all $\rho \in \mathbb{R}^{+}$, and $\|\cdot\|$ is the Euclidean norm. The functions $p$ and $f$ can be thought of as a bounded perturbation and a locally bounded deformation, respectively. The constant $K_{p}$ is a perturbation bound, and the functions $h_{L}$ and $h_{U}$ bound the amount of local deformation. Observe that outside of points of occlusion, this model is able to describe the transformation between pairs of views of the same scene or the evolution of a deforming object. An example of such deformation functions are:

$$
h_{L}(\rho)=\left(1-K_{d}\right) \rho \quad \text { and } \quad h_{U}(\rho)=\left(1+K_{d}\right) \rho
$$

which corresponds to a Lipschitz deformation model with deformation constant $K_{d}$. This bounding function requires that deformations are bounded linearly with respect to the distance between points in an image. Throughout the rest of the paper, we assume that the images $I_{0}$ and $I_{1}$ satisfy the deformation model specified in Equations (1) and (2), where the perturbation bound $K_{p}$ and the bounding deformation functions $h_{L}, h_{U}$ are known. However, the actual perturbation $p(x)$ and deformation $f(x)$ functions are unknown.

Next, we describe explicitly the two problems we attempt to address using our descriptor.

Problem 1. Given an arbitrary point $x_{0} \in \Omega$ and a set of points $\bar{\Lambda}_{1} \subset \Omega$, find the point $x_{1} \in \bar{\Lambda}_{1}$ such that:

$$
\left\|f^{-1}\left(x_{0}\right)-x_{1}\right\|=\min _{x \in \bar{\Lambda}_{1}}\left\|f^{-1}\left(x_{0}\right)-x\right\| .
$$

The solution to this problem has direct implications for wide baseline matching and image retrieval. When $\bar{\Lambda}_{1}$ is taken as uniform grid of points, we call the solution to Problem 1 a grid matching point. Denoting the area of the set $E$ by $|E|$ and defining $B(x, r)=\{y \in \Omega \mid\|x-y\| \leq r\}$, our second problem is related to the first but attempts to explicitly identify neighborhoods rather than points with sufficient overlap:

Problem 2. Given a threshold $\tau \in[0,1]$, called the overlap threshold, neighborhoods $B\left(x, R_{0}\right) \subset \Omega$ and $B\left(x^{\prime}, R_{1}\right) \subset$ $\Omega$ for some $x, x^{\prime} \in \Omega$ and $R_{0}, R_{1}>0$, is

$$
\frac{\left|f^{-1}\left(B\left(x, R_{0}\right)\right) \cap B\left(x^{\prime}, R_{1}\right)\right|}{\left|f^{-1}\left(B\left(x, R_{0}\right)\right) \cup B\left(x^{\prime}, R_{1}\right)\right|}>\tau ?
$$


Note that the above quantity corresponds to the ratio between the area in the intersection and the union of the pair of neighborhood after mapping to the domain of $I_{1}$. This quantity is directly related to determining region correspondences which has important applications for object recognition and registration.

\section{Set Filtrations}

In this section, we introduce the results necessary to robustly characterize a neighborhood of a point in terms of topology. We begin by describing several results from algebraic topology, most importantly the homology group of a set. We then describe how our deformation model dictates the allowed transformations of the homologies for the pair of images. We conclude the section by strengthening the results on the transformation of homologies to local neighborhoods of the image. The proofs of all of the results included in this section can be found in a technical report [6].

\subsection{Background}

The objective of this section is to give a brief overview of algebraic topology. A comprehensive introduction to these ideas can be found in Chapter 2 of [3]. Algebraic topology explicitly characterizes the properties of spaces that are preserved under continuous deformation in terms of algebraic objects. Homology theory in particular transforms the study of topological invariants into the study of groups. If, for example, one wants to determine whether a pair of spaces are homeomorphic, one can transform the problem into determining whether a pair of groups are equivalent. In fact, by comparing the rank of the pair of groups, which is equal to the number of basis elements required to generate the group, one can effectively determine whether the pair of spaces are homeomorphic.

Naïvely comparing pairs of homology is generally insufficient to perform matching between pairs of images transforming under a homeomorphism for two reasons. First, though pairs of images maybe transforming under a homeomorphism the effect of digitization (especially along edges) can ruin the applicability of homology. To address this deficiency, we define conditions on the homology over processed images. Second, homology is too coarse a construct. This is due to the fact that comparison between the homologies of different spaces is done via a counting argument and because homology is generally defined over entire spaces. We address homologies coarseness by localizing homology over intensity and space.

To understand these various extensions of homology, we must begin by describing homology more explicitly. The 0 -homology, denoted $H_{0}(E)$, is a group whose rank is equal to the number of connected components in the space $E$. Whereas, the 1-homology, denoted $H_{1}(E)$, is a group whose rank is equal to the number of distinct cycles in the space $E$ that cannot be shrunk via continuous deformation to a single point. Generalizing this notion let each of the $k$ homology groups of the space $E$ be denoted $H_{k}(E)$. Suppose that one is given a map $\sigma: E_{1} \rightarrow E_{2}$ between two spaces, we can in fact determine how topological properties transform under $\sigma$ by considering the homomorphism (this generalizes the notion of a linear map to groups) induced by $\sigma$ denoted $\sigma *: H_{k}\left(E_{1}\right) \rightarrow H_{k}\left(E_{2}\right)$. The case when $E_{1} \subset E_{2}$ and $\sigma$ is the inclusion map is called a filtration and is particularly important. To illustrate its utility consider the following result:

Lemma 1. Given the filtration $E_{1} \subset E_{2} \subset E_{3} \subset E_{4}$ and inclusion maps $\sigma_{i, j}: E_{i} \rightarrow E_{j}$ where $i<j$, then

$$
\operatorname{rank}\left(\frac{H_{k}\left(E_{1}\right)}{\operatorname{ker} \sigma_{1,4} *}\right) \leq \operatorname{rank}\left(\frac{H_{k}\left(E_{2}\right)}{\operatorname{ker} \sigma_{2,3^{*}}}\right), \quad \forall k \geq 0,
$$

where ker computes the kernel of its argument.

This result gives a straightforward method to quantify the topological structure that must by carried from $E_{2}$ to $E_{3}$ by analyzing the structure carried from $E_{1}$ to $E_{4}$. Importantly, notice that neither of the mappings from $E_{1}$ to $E_{2}$ or from $E_{3}$ to $E_{4}$ are needed in this result. In the next few subsections, we describe how this result can be used to extend homology to address its aforementioned deficiencies.

\subsection{Global Filtration}

Let us begin by defining a set of pre-processed images.

Definition 1. Let the pre-processed images be defined as:

$$
\begin{aligned}
I_{i--}(x) & =\inf _{y \in B\left(x, g_{i}(\rho)\right)} I_{i}(y) \\
I_{i-}(x) & =\inf _{y \in B(x, \rho)} I_{i}(y) \\
I_{i+}(x) & =\sup _{y \in B(x, \rho)} I_{i}(y) \\
I_{i++}(x) & =\sup _{y \in B\left(x, g_{i}(\rho)\right)} I_{i}(y)
\end{aligned}
$$

for $i \in\{0,1\}$, where $\rho \geq 0$ and

$$
g_{i}(\rho)= \begin{cases}h_{U}(\rho) & \text { if } i=0 \\ h_{L}^{-1}(\rho) & \text { if } i=1\end{cases}
$$

We define the following inter-level sets:

$$
\begin{aligned}
E_{i--} & =I_{i-}^{-1}\left[a+K_{p}, \infty\right) \cap I_{i++}^{-1}\left(-\infty, b-K_{p}\right] \\
E_{i-} & =I_{i-}^{-1}[a, \infty) \cap I_{i+}^{-1}(-\infty, b] \\
E_{i+} & =I_{i+}^{-1}[a, \infty) \cap I_{i-}^{-1}(-\infty, b] \\
E_{i++} & =I_{i++}^{-1}\left[a-K_{p}, \infty\right) \cap I_{i--}^{-1}\left(-\infty, b+K_{p}\right]
\end{aligned}
$$

for constants $a$ and $b$ such that $b-a>2 K_{p}$.

The inter-level sets are the objects upon which we perform homology computation and help us localize homology over each image's intensity space. Fig. 2 illustrates these pre-processed images. Their corresponding inter-level sets are drawn in Fig. 3. The inter-level sets satisfy certain properties: 

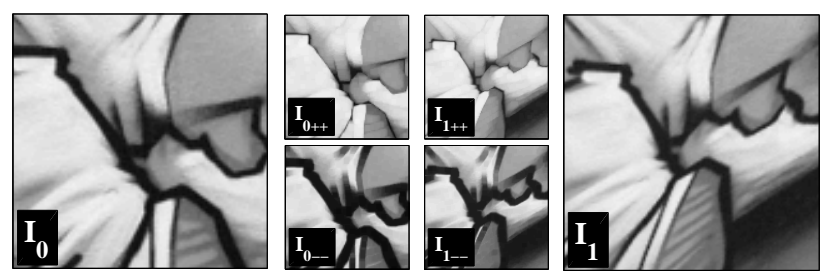

Figure 2. Pre-processed Images. Original images $I_{0}$ (left) and $I_{1}$ (right). Illustration of several pre-processed images (middle). These pre-processed images are defined to compensate for digitization effects along edges.

Lemma 2. The inter-level sets for any $a$ and $b$ such that $b-a>2 K_{p}$ satisfy:

$$
\begin{aligned}
& E_{0--} \subset f\left(E_{1-}\right) \subset f\left(E_{1+}\right) \subset E_{0++} \\
& f\left(E_{1--}\right) \subset E_{0-} \subset E_{0+} \subset f\left(E_{1--}\right)
\end{aligned}
$$

The result of the previous lemma ensures our choice of pre-processed images allows us to define a filtration between sets in images 0 and 1 . As a result of Lemma 1, we obtain the following result:

Theorem 1. Given images $I_{0}$ and $I_{1}$, and constants $a, b$ such that $b-a>2 K_{p}$, then

$$
\operatorname{rank}\left(\frac{H_{k}\left(E_{i--}\right)}{\operatorname{ker} \sigma_{i--, i++^{*}}}\right) \leq \operatorname{rank}\left(\frac{H_{k}\left(E_{j-}\right)}{\operatorname{ker} \sigma_{j-, j+*}}\right)
$$

where $i, j \in\{0,1\}$ such that $j \neq i$, and $\sigma_{i--, i++}$ : $E_{i--} \rightarrow E_{i++}$ and $\sigma_{j-, j+}: E_{j-} \rightarrow E_{j+}$ are the inclusion maps.

This theorem gives a computable condition in terms of the ranks of homologies that must be satisfied by the corresponding inter-level sets defined by image 0 and 1 . To understand this result, observe that the rank of $\frac{H_{0}\left(E_{i-}\right)}{\operatorname{ker} \sigma_{i-, i+*}}$ is equal to the number of connected components in $E_{i+}$ that have a non-empty intersection with $E_{i-}$. This can be understood as the number of components that persist from $E_{i-}$ to $E_{i+}$. Hence, this theorem tells us that the number of connected components that persist from $E_{1--}$ to $E_{1++}$ is less

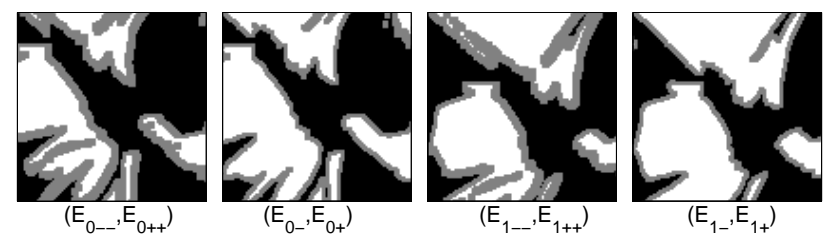

Figure 3. Inter-level sets for images 0 and 1 from Fig. 2. The left panel shows the set $E_{0--}$ in white, and the set $E_{0++}$ in gray. The other plots follow the same labeling convention. As stated by Theorem 1, $\operatorname{rank}\left(\frac{H_{0}\left(E_{1--}\right)}{\operatorname{ker} \sigma_{1--, 1++}}\right)=3$ (i.e. the number of connected components that persist from $E_{1--}$ to $E_{1++}$ ) which is less than $\operatorname{rank}\left(\frac{H_{0}\left(E_{0-}\right)}{\operatorname{ker} \sigma_{0-, 0+^{*}}}\right)=5$. than the number of components that persist from $E_{0-}$ to $E_{0+}$. If this condition is violated for a pair of corresponding inter-level sets, then the pair of images that were used to construct these inter-level sets do not satisfy our image model. Observe that by defining the inter-level sets on preprocessed images, we avoid the problem of digitization effects along edges. An example illustrating an application of this theorem can be found in Fig. 3 .

\subsection{Spatially Localized Filtrations}

The previous subsection gave a topological method to robustly determine whether entire images satisfied our deformation model. In this subsection, we define a spatial localization of this result. In order to obtain a localized characterization of an image, we begin by letting $\Lambda_{1}=\left\{x_{1, i j}\right\} \subset$ $\Omega$ denote a uniform grid of points spaced $s_{1}$ units apart. Our objective in this subsection is to construct a local set of conditions similar to those described in Theorem 1 that must be satisfied by a point in $\Lambda_{1}$ that is a solution to Problem 1, i.e. a grid matching point.

First, we obtain neighborhoods around a point $x_{0}$ and its grid matching point $x_{1} \in \Lambda_{1}$ that satisfy a sequence of inclusions.

Lemma 3. Given a point $x_{0} \in \Omega$, a corresponding grid matching point $x_{1}$, radius $r_{0}>h_{U}\left(s_{1} / \sqrt{2}\right)$, and radius $r_{1}>h_{L}^{-1}\left(h_{U}\left(s_{1} / \sqrt{2}\right)\right)$, then

$$
B\left(x_{0}, r_{0-}\right) \subset f\left(B\left(x_{1}, r_{1}\right)\right) \subset B\left(x_{0}, r_{0+}\right)
$$

and

$$
B\left(x_{1}, r_{1-}\right) \subset f^{-1}\left(B\left(x_{0}, r_{0}\right)\right) \subset B\left(x_{1}, r_{1+}\right)
$$

where

$$
\begin{aligned}
& r_{1-}=h_{U}^{-1}\left(r_{0}-h_{U}\left(s_{1} / \sqrt{2}\right)\right) \\
& r_{0-}=h_{L}\left(r_{1}\right)-h_{U}\left(s_{1} / \sqrt{2}\right) \\
& r_{0+}=h_{U}\left(r_{1}\right)+h_{U}\left(s_{1} / \sqrt{2}\right) \\
& r_{1+}=h_{L}^{-1}\left(r_{0}+h_{U}\left(s_{1} / \sqrt{2}\right)\right)
\end{aligned}
$$

As before, the inclusions in the previous lemma define a filtration in terms of inter-level sets.

Definition 2. Let the $\left(r_{0}, r_{1}\right)$-localized inter-level sets between a point $x_{0} \in \Omega$ and its grid matching point $x_{1}$ be defined as

$$
\begin{aligned}
E_{i--}\left(x_{i}\right) & =E_{i--} \cap B\left(x_{i}, r_{i-}\right) \\
E_{i-}\left(x_{i}\right) & =E_{i-} \cap B\left(x_{i}, r_{i}\right) \\
E_{i+}\left(x_{i}\right) & =E_{i+} \cap B\left(x_{i}, r_{i}\right) \\
E_{i++}\left(x_{i}\right) & =E_{i++} \cap B\left(x_{i}, r_{i+}\right)
\end{aligned}
$$

for $i \in\{0,1\}, r_{0}>h_{U}\left(s_{1} / \sqrt{2}\right), r_{1}>h_{L}^{-1}\left(h_{U}\left(s_{1} / \sqrt{2}\right)\right)$. The radii $r_{i-}$ and $r_{i+}$ are given as in Lemma 3 . 
Note that the $\left(r_{0}, r_{1}\right)$-localized inter-level sets satisfy inclusion relations similar to the ones stated in Lemma 2 by construction. Hence, we have the following localized counterpart to Theorem 1:

Theorem 2. Given images $I_{0}$ and $I_{1}$, constants $a, b$ such that $b-a>2 K_{p}$, and radii $r_{0}>h_{U}\left(s_{1} / \sqrt{2}\right)$ and $r_{1}>$ $h_{L}^{-1}\left(h_{U}\left(s_{1} / \sqrt{2}\right)\right)$, then for any $x_{0} \in \Omega$ and grid matching point $x_{1} \in \Lambda_{1}$ we have that

$$
\operatorname{rank}\left(\frac{H_{k}\left(E_{i--}\left(x_{i}\right)\right)}{\operatorname{ker} \sigma_{i--, i++^{*}}}\right) \leq \operatorname{rank}\left(\frac{H_{k}\left(E_{j-}\left(x_{j}\right)\right)}{\operatorname{ker} \sigma_{j-, j+} *}\right)
$$

where $i, j \in\{0,1\}$ such that $j \neq i$, and $\sigma_{i--, i++}$ : $E_{i--}\left(x_{i}\right) \rightarrow E_{i++}\left(x_{i}\right)$ and $\sigma_{j-, j+}: E_{j-}\left(x_{j}\right) \rightarrow$ $E_{j+}\left(x_{j}\right)$ are the natural inclusion maps.

This result gives a way to identify the existence of a local homeomorphism between neighborhoods around $x_{0}$ and $x_{1}$. That is, if these conditions are not satisfied, then $x_{1}$ cannot be a point that corresponds to $x_{0}$ under our image model.

\section{Topological Attributed Relational Graph}

At this point, we in fact have a robust localized topological descriptor that could be used to perform matching. In this section, we describe how to construct a graphical representation that integrates the localized topological characterization developed so far to render the constructed descriptor more distinct.

Suppose as in the previous section, we have a grid of points $\Lambda_{1}$ placed $s_{1}$ units apart, a set of radii and constants $\left\{\left(r_{0, \lambda}, a_{\lambda}, b_{\lambda}\right)\right\}_{\lambda \in \Gamma}$ that satisfy the conditions in Theorem 2 where $\Gamma$ is some indexing set, and let

$$
r_{1, \lambda}=\left(h_{L}^{-1}\left(r_{0, \lambda}\right)+h_{U}^{-1}\left(r_{0, \lambda}\right)\right) / 2
$$

Let us also assume a uniform grid of points $\Lambda_{0}=\left\{x_{0, \gamma}\right\} \subset$ $\Omega$ spaced $s_{0}>2 h_{U}\left(s_{1} / \sqrt{2}\right)$ units apart, and let $\left\{x_{1, \gamma}\right\} \subset$ $\Lambda_{1}$, be the set of corresponding grid matching points. The following theorem gives a set of bounds between the distance of points in $\Lambda_{0}$ and grid matching points in $\Lambda_{1}$ :

Theorem 3. Given the pair of point $\left(x_{0, \alpha}, x_{0, \beta}\right) \in \Lambda_{0} \times \Lambda_{0}$ and corresponding matching points $\left(x_{1, \alpha}, x_{1, \beta}\right) \in \Lambda_{1} \times \Lambda_{1}$, then

$$
h_{U}^{-1}\left(\left\|x_{0, \alpha}-x_{0, \beta}\right\|-2 h_{U}\left(s_{1} / \sqrt{2}\right)\right) \leq\left\|x_{1, \alpha}-x_{1, \beta}\right\|
$$

and

$$
\left\|x_{1, \alpha}-x_{1, \beta}\right\| \leq h_{L}^{-1}\left(\left\|x_{0, \alpha}-x_{0, \beta}\right\|+2 h_{U}\left(s_{1} / \sqrt{2}\right)\right) .
$$

For each of the points $x_{0, \gamma}$ and corresponding grid matching points $x_{1, \gamma}$, the conditions specified by Theorem 2 must be satisfied by all tuples $\left(r_{0, \lambda}, r_{1, \lambda}, a_{\lambda}, b_{\lambda}\right)$. Additionally, for each pair $\left(x_{0, \alpha}, x_{0, \beta}\right) \in \Lambda_{0} \times \Lambda_{0}$ and corresponding grid matching pair $\left(x_{1, \alpha}, x_{1, \beta}\right) \in \Lambda_{1} \times \Lambda_{1}$ the distance bounds in Theorem 3 must be satisfied.

To check the simultaneous satisfaction of all of these conditions, we can recast our problem by constructing a Topological-Attributed Relational Graph or T-ARG, $\mathcal{G}_{0}$ as follows: let the nodes of this graph represent the points $x_{0, \gamma}$ and label these nodes with the rank conditions defined in Theorem 2 and let the edges of this graph represent the distance between points and be labeled using the distance bounds from Theorem 3. We can construct a similar TARG, $\mathcal{G}_{1}$, using the points $x_{1, \gamma}$. In fact, we can define a T-ARG $\hat{\mathcal{G}}_{1}$ using all the points in $\Lambda_{1}$, the results of the Theorems 2 and 3 give constraints to determine a subgraph isomorphism from $\mathcal{G}_{0}$ to $\mathcal{G}_{1} \subset \hat{\mathcal{G}}_{1}$. More explicitly a correspondence between the graph $\mathcal{G}_{0}$ and a subgraph in $\hat{\mathcal{G}}_{1}$ is defined by the satisfaction of Theorems 2 and 3 . Hence, the identification of $\mathcal{G}_{1}$ turns into the matching of Attributed Relational Graphs for which the attributes are topological rank conditions and distance bounds.

\section{Implementation}

In this section, we begin by explicitly describing how we can solve the problems defined in Section 2 using the TARG. We also describe how we implement our algorithm. The executables used to perform this implementation can be found online ${ }^{1}$.

Solution 1. Given an arbitrary set of points $\bar{\Lambda}_{1}$, we can first construct a grid of points $\Lambda_{1}$ placed $s_{1}$ units apart such that $\bar{\Lambda}_{1} \subset \Lambda_{1}$. Then given a point $x \in \Omega$ and another point $x^{\prime} \in$ $\Lambda_{1}$, we identify $x^{\prime}$ as a possible grid matching point if there is a subgraph isomorphism from a graph $\mathcal{G}_{0}$ into a graph $\hat{\mathcal{G}}_{1}$. $\mathcal{G}_{0}$ is constructed using the points in $\Lambda_{0} \cap B\left(x, R_{0}\right)$, and $\hat{\mathcal{G}}_{1}$ uses the points in $\Lambda_{1} \cap B\left(x^{\prime}, R_{1}\right)$, where $R_{1}=h_{L}^{-1}\left(R_{0}+\right.$ $2 h_{U}\left(s_{1} / \sqrt{2}\right)$ ) and $R_{0}>0$. It is then straightforward to re-project the solution in $\Lambda_{1}$ to the solution in $\bar{\Lambda}_{1}$.

Note that $R_{1}$ is defined using Theorem 3 and $R_{0}$ is set arbitrarily based on how large of a neighborhood around $x$ we are interested in considering.

To solve Problem 2, we are interested in determining the overlap ratio between sets $f^{-1}\left(B\left(x, R_{0}\right)\right)$ and $B\left(x^{\prime}, R_{1}\right)$. Note that if $x_{1}$ is a grid matching point to $x$, then:

$$
B\left(x_{1}, R_{1-}\right) \subset f^{-1}\left(B\left(x, R_{0}\right)\right) \subset B\left(x_{1}, R_{1+}\right)
$$

where $R_{1-}$ and $R_{1+}$ are defined as in Lemma 3. Using Solution 1 , we obtain a set of possible grid matching points to $x$. An upper bound to the overlap ratio between $B\left(x, R_{0}\right)$ and $B\left(x^{\prime}, R_{1}\right)$ can be computed by considering the maximum of the bounds using all possible grid matches.

\footnotetext{
1 http://people.engr.ncsu.edu/ejlobato/Research/ 2011/FeatureMatching/
} 


\begin{tabular}{l|l}
\hline Parameter & Description \\
\hline$K_{p}$ & $\begin{array}{l}\text { Perturbation bound. A value of } 5 \text { is used. } \\
K_{d}\end{array}$ \\
& $\begin{array}{l}\text { Deformation constant. A value of } 0.1 \text { is } \\
\text { used unless otherwise specified. }\end{array}$ \\
\hline & Table 1. Image Model Parameters
\end{tabular}

Solution 2. Given neighborhoods $B\left(x, R_{0}\right)$ and $B\left(x^{\prime}, R_{1}\right)$ where $x, x^{\prime} \in \Omega$, we identify a possible matching set with overlap greater than $\tau$ if

$$
\frac{\left|B\left(x_{1}, R_{1+}\right) \cap B\left(x^{\prime}, R_{1}\right)\right|}{\left|B\left(x_{1}, R_{1-}\right) \cup B\left(x^{\prime}, R_{1}\right)\right|}>\tau,
$$

where $x_{1}$ is the possible grid matching point to $x$ that is closest to $x^{\prime}$.

In our implementation we construct our topological descriptors using the rank of the 0-homology group. Recall that the 0-homology corresponds to the connected components. For example, the rank of $\frac{H_{k}\left(E_{i-}\right)}{\operatorname{ker} \sigma_{i-, i+*}}$ is equal to the number of connected components in $E_{i+}$ that have a nonempty intersection with $E_{i-}$. In our implementation we assume the Lipschitz deformation model defined in Equation 3. All the parameters required for our method are outlined in Tables 1 and 2. Note that the image model parameters are the only pieces of information required about the image. The algorithmic parameters (i.e. Table 2) on the other hand represent parameters in our algorithm and do not affect the validity of the approach.

\section{Results}

In this section, we describe our performance on a dataset constructed from standard benchmark images. We analyze the performance of our descriptor by computing its precision and recall as in [11]. To determine a ground truth, two

\begin{tabular}{l|l}
\hline Parameter & Description \\
\hline$\rho$ & $\begin{array}{l}\text { Radius of morphological operator for pre- } \\
\text { processing. Value set to } 2 .\end{array}$ \\
$s_{0}$ & $\begin{array}{l}\text { Spacing for grid } \Lambda_{0} . \text { Value set to } 6 . \\
\text { Spacing for grid } \Lambda_{1} . \text { Value set to } 2 .\end{array}$ \\
$s_{1}$ & $\begin{array}{l}\left.r_{0, \lambda}, a_{\lambda}, b_{\lambda}\right) \\
\text { Radii and constant used to define the } \\
\text { topological rank descriptors. We use } \\
\text { all combination such that } r_{0, \lambda} \in \\
\{2,3,4,6,9,14,19,24,30\}, \text { and }\left[a_{\lambda}, b_{\lambda}\right] \\
\text { corresponds to any of the intervals ob- } \\
\text { tained from partitioning the range }[0,255] \\
\text { into } 4,8 \text { and } 16 \text { evenly spaced intervals. } \\
\text { Radius of neighborhood around } x_{0} \text { used } \\
\text { to construct graph } \mathcal{G}_{0} \text { in Solution 1. A } \\
\text { value of 30 is used. }\end{array}$ \\
\hline Table 2. Algorithmic Parameters
\end{tabular}

regions are said to match if their overlap ratio is greater than a specified threshold $\tau$. Precision and recall are defined as:

$$
\text { Precision }=\frac{\# \text { of correct matches by algorithm }}{\# \text { of total detections by algorithm }}
$$

and

$$
\text { Recall }=\frac{\# \text { of correct matches by algorithm }}{\# \text { of total true matches }} .
$$

In contrast to other descriptors, T-ARG does not employ a distance function to compare feature vectors. Rather, either a feature satisfies the conditions of Theorems 2 and 3, in which case it matches, or it does not satisfy those conditions, in which case it does not match. However, as described in Section 5, we construct bounds for the overlap given our choice of parameters. By thresholding our estimated bounds on the overlap using a parameter $\tau_{\text {top }}$, we can get a precision/recall curve parameterized by the value of this parameter as other descriptors do with their appropriately defined distance function. The time required to construct a descriptor for a single region is approximately 20 s on a 2.2 Dual-Core i7 CPU with 8 GB of memory. For our method, we set the parameters as described in Tables 1 and 2.

\subsection{General Deformation Images}

In order to analyze the performance of our approach in the presence of deformations we construct two synthetic datasets: Graffiti and Boat. The Graffiti images are of size $800 \times 640$ and the Boat images are $850 \times 680$. We consider a controlled perturbation using the function $f: \Omega \rightarrow \Omega$ given by:

$$
f^{-1}\left(\begin{array}{l}
z_{1} \\
z_{2}
\end{array}\right)=\left(\begin{array}{l}
z_{1}+0.5 c \cos \left(0.02 z_{2}\right) \\
z_{2}+0.5 c \cos \left(0.02 z_{1}\right)
\end{array}\right)
$$

where the factor $c$ specifies a maximum deformation factor (e.g. $c=10$ indicates a maximum deformation of $10 \%$ ). We also study the effect of noise by adding a random uniform perturbation to the images with magnitude equal to 10 . Examples from our dataset are illustrated in Fig. 1 and 4. A subset of regions from the images to be matched are chosen

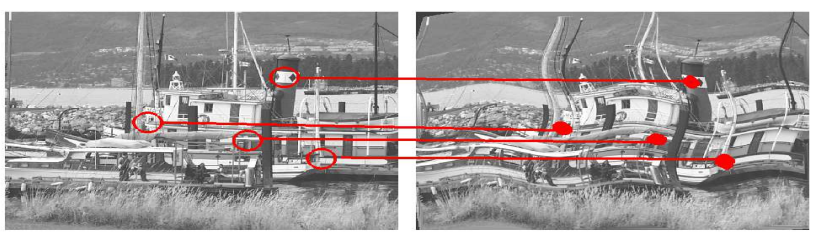

Figure 4. Point Matching Samples: The base image from the Boat dataset (left) with corresponding neighborhoods $B\left(x, R_{0}\right)$. Corresponding matches found on an image showing a $30 \%$ deformation, i.e. $c=30$ in Equation 24 (right). 

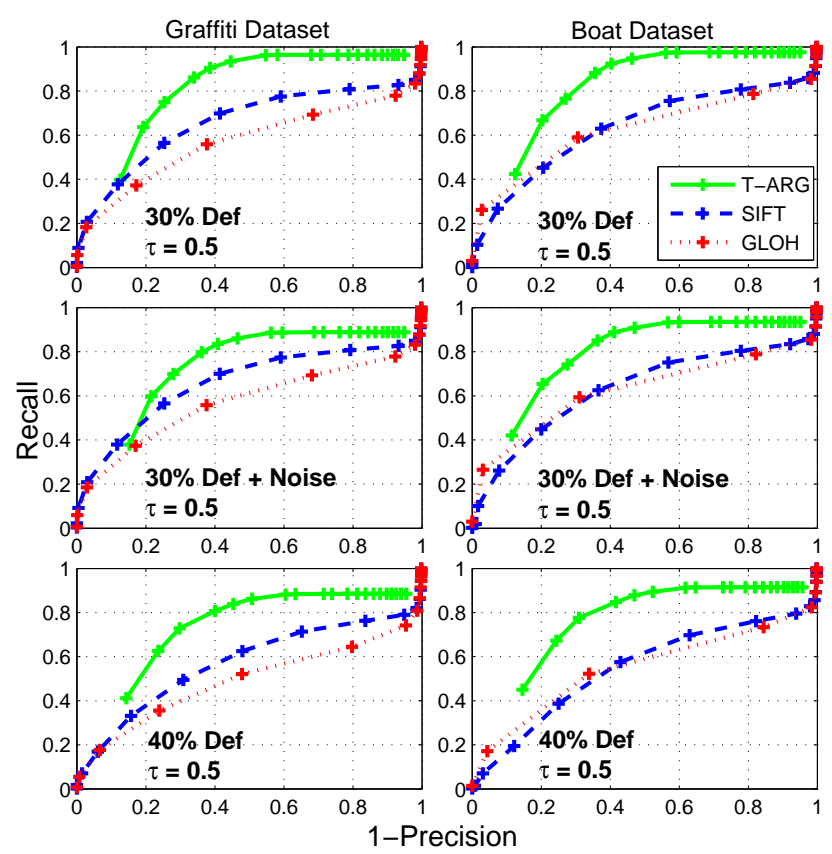

Figure 5. Precision and Recall $(\tau=0.5)$ : Results for the Graffiti (left) and Boat (right) datasets. Results for image with 30\% deformation (top), $30 \%$ deformation and uniform random noise in the interval $[-10,10]$ (middle), and $40 \%$ deformation (bottom).

to test. We select circular regions of radius 30 from both images centered around points from a uniform grid of points spaced 12 units from each other while removing the points that are 60 units from the boundary of the image. This gives around 2500 regions for each image in the datasets.

Fig. 5 shows the comparison of our approach to SIFT and GLOH for a ground truth threshold of $\tau=0.5$. The first row corresponds to comparing the base images from the Graffiti and Boat datasets against an image with 30\% deformation and no noise. The second row corresponds to the base image being matched to an image with $30 \%$ deformation and with uniform noise in the interval $[-10,10]$ added to each pixel. The final row corresponds to the base image being matched to an image constructed with $40 \%$ deformation and no noise. As expected from our method, we obtain a very high recall rate since our derivations attempt to avoid false-negatives. Observe that these results are obtained using an estimated deformation value of $10 \%$ (i.e. $K_{d}=10$ ) which is far less than the actual maximal deformation for the images. Note that adding uniform random noise to an image (as shown in the middle row) and increasing the deformation (as shown in the bottom row) have little effect on the performance of our approach.

Fig. 6 illustrates similar results for a ground truth threshold of $\tau=0.2$. In terms of the overlap ratio, this corresponds to treating pairs with less actual overlap as potential matches. In this case, the performance of our algorithm im-

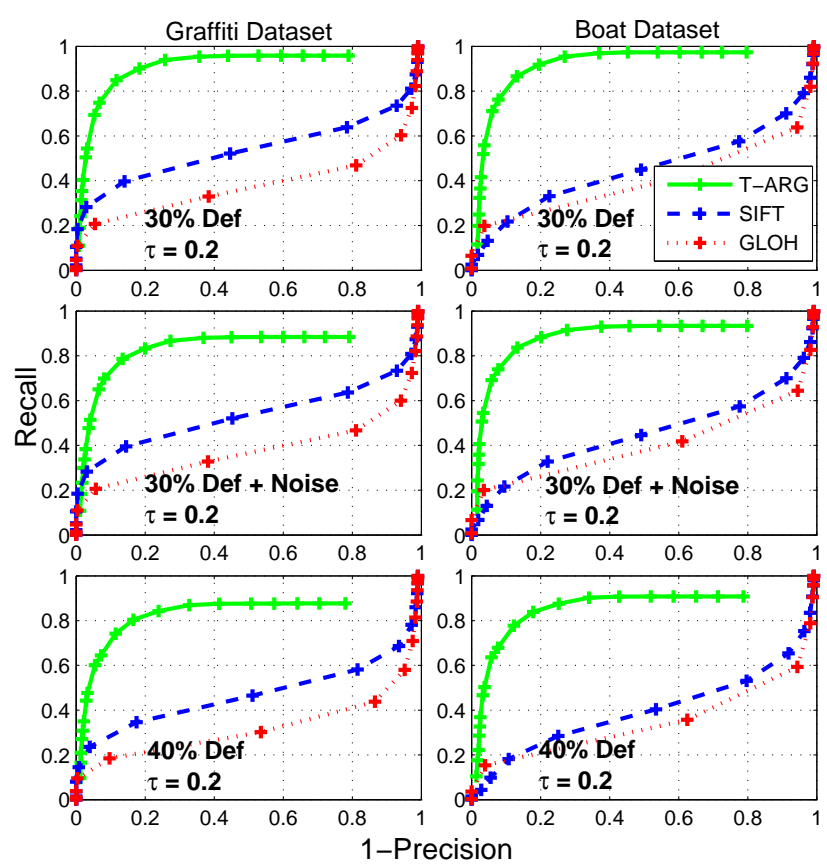

Figure 6. Precision and Recall $(\tau=0.2)$ : Results for the Graffiti (left) and Boat (right) datasets. Results for image with $30 \%$ deformation (top), $30 \%$ deformation and uniform random noise in the interval $[-10,10]$ (middle), and $40 \%$ deformation (bottom).

proves even though the performance of the SIFT and GLOH descriptors decreases. Finally, Fig. 7 illustrates the dependency of our approach on the choice of parameter $K_{d}$ for the images with $30 \%$ deformation. We choose a ground truth threshold of $\tau=0.5$ and an overlap threshold for our algorithm of $\tau_{t o p}=\tau / 3$. The plot illustrates the precision and recall for our algorithm against SIFT and GLOH as we change the value of $K_{d}$ from 0.05 to 0.20 .

\subsection{Homography Images}

Next, we analyze the performance of our approach by constructing a new dataset, called the Homography Graffiti Dataset, by applying a homography to the left image

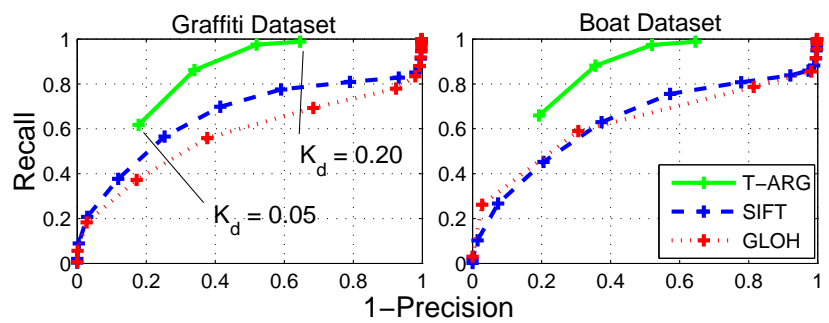

Figure 7. Dependency on deformation bound $K_{d}$. Results for Graffiti (left) and Boat (right) datasets as we change $K_{d}$ from 0.05 to 0.20 . The ground truth overlap threshold is set to $\tau=0.5$. The overlap threshold for our algorithm is set to $\tau_{\text {top }}=\tau / 3$. 


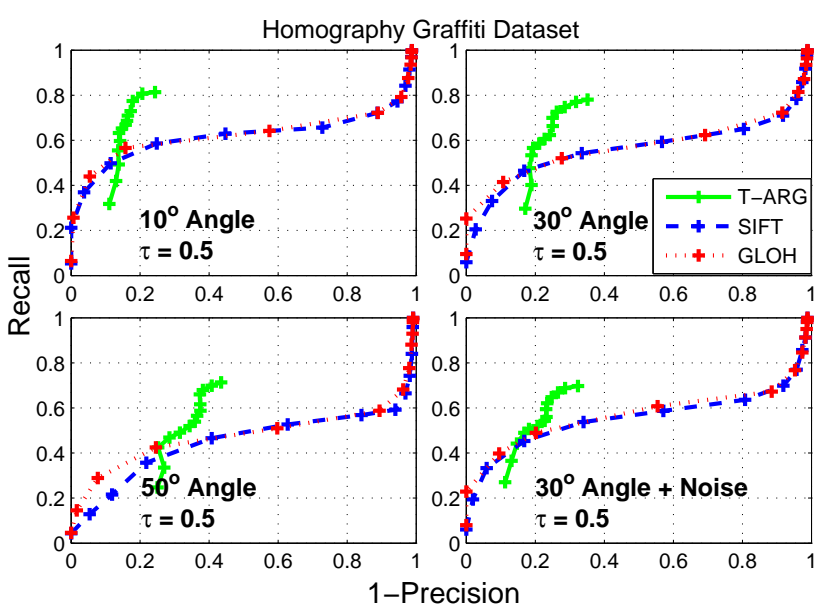

Figure 8. Precision and Recall ( $\tau=0.5$ ): Results for the Homography Graffiti Dataset. Results for image with $10^{\circ}$ change in viewing angle (top left), $30^{\circ}$ change in viewing angle (top right), $50^{\circ}$ change in viewing angle (bottom left), and $30^{\circ}$ change in viewing angle and uniform random noise in the interval $[-10,10]$ (bottom right).

of Fig. 1 corresponding to a change in viewing angle by $10^{\circ}, 30^{\circ}, 50^{\circ}$, and $30^{\circ}$ with uniform noise in the interval $[-10,10]$ added to each pixel.

In order to give SIFT and GLOH an advantage we select 200 regions by using the Harris Affine Region Detector with a threshold of 80,000 [11]. We determine the overlap estimate between two regions by first normalizing each detected region into a circular region of radius 30 and then apply our algorithm. Fig. 8 shows the comparison for a ground truth threshold of $\tau=0.5$. Notice that regardless of choosing regions specifically preferred by SIFT and GLOH upon which to compare the performance of our matching approach on, our method still outperforms the traditional methods.

\section{Conclusion}

In this paper, we introduced T-ARG, a new local photometric descriptor that can effectively perform deformation invariant image matching. T-ARG is a robust topological descriptor backed by a formal mathematical framework. We applied T-ARG to a set of standard benchmark images with applied deformations and perturbations and demonstrated that T-ARG significantly outperforms traditional descriptors. In the immediate future we plan to apply the method to naturally deforming scenes such as animals, cloth, and medical images.

The utility of our approach is that it generalizes in a straightforward manner the comparison of multidimensional datasets undergoing bounded deformations. There is a deep relationship between the bounds on the rank of homology presented in Section 3 and the theory of Persis- tent Topology [14]. In the future we look forward to being able to employ the computational tools available to compute persistence to speed up our implementation and help build an even more powerful topological descriptor. Other potential extensions of this work include: (1) its generalization to account for larger lighting variations present in natural images, and (2) the development of a region detector to determine proper choice of scale for regions of interest.

\section{References}

[1] S. Belongie, J. Malik, and J. Puzicha. Shape Matching and Object Recognition Using Shape Contexts. IEEE Transactions on Pattern Analysis and Machine Intelligence, 24(4):509-522, 2002. 2

[2] W. Freeman and E. Adelson. The design and use of steerable filters. IEEE Transactions on Pattern Analysis and Machine Intelligence, 13(9):891-906, 2002. 2

[3] A. Hatcher. Algebraic Topology. Cambridge University, 2002. 3

[4] A. Johnson and M. Hebert. Using Spin Images for Efficient Object Recognition in Cluttered 3D Scenes. IEEE Transactions on Pattern Analysis and Machine Intelligence, 21(5):433-449, 2002. 2

[5] H. Ling and D. Jacobs. Deformation Invariant Image Matching. In International Conference on Computer Vision 2005, pages 1466-1473, 2005. 2

[6] E. Lobaton, R. Vasudevan, R. Alterovitz, and R. Bajcsy. Robust Topological Features for Deformation Invariant Image Matching. Technical Report UCB/EECS-2011-89, EECS Department, University of California, Berkeley, Aug 2011. 3

[7] E. Lobaton, R. Vasudevan, R. Bajcsy, and R. Alterovitz. Local Occlusion Detection under Deformations Using Topological Invariants. In European Conference on Computer Vision 2010, pages 101-114, 2010. 2

[8] D. Lowe. Object Recognition from Local Scale-Invariant Features. In International Conference on Computer Vision 1999, pages 1150-1157, 1999. 1

[9] D. Lowe. Distinctive Image Features from Scale-Invariant Keypoints. International Journal of Computer Vision, 60(2):91-110, 2004. 2

[10] K. Mikolajczyk and C. Schmid. Indexing Based on Scale Invariant Interest Points. In International Conference on Computer Vision 2001, pages 525-531, 2002. 1

[11] K. Mikolajczyk and C. Schmid. A Performance Evaluation of Local Descriptors. IEEE Transactions on Pattern Analysis and Machine Intelligence, 27(10):1615-1630, 2005. 2, 6, 8

[12] F. Schaffalitzky and A. Zisserman. Multi-View Matching for Unordered Image Sets. European Conference on Computer Vision 2002, pages 414-431, 2002. 2

[13] T. Tuytelaars and L. Van Gool. Matching Widely Separated Views Based on Affine Invariant Regions. International Journal of Computer Vision, 59(1):61-85, 2004. 1

[14] A. Zomorodian and G. Carlsson. Computing Persistent Homology. Discrete and Computational Geometry, 33(2):249274, 2005. 8 